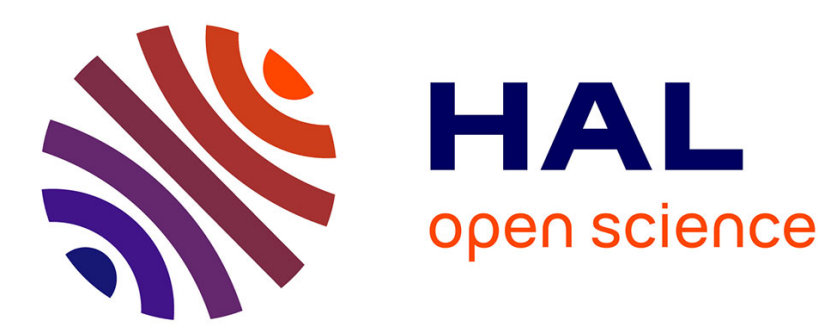

\title{
Bending modulus of bidisperse particle rafts: Local and collective contributions
}

\author{
Pauline C. Petit, Anne-Laure Biance, Carole Planchette, Elise Lorenceau
}

\section{To cite this version:}

Pauline C. Petit, Anne-Laure Biance, Carole Planchette, Elise Lorenceau. Bending modulus of bidisperse particle rafts: Local and collective contributions. Physical Review E , 2016, 93 (4), pp.2361 2376. 10.1103/PhysRevE.93.042802 . hal-01611595

\section{HAL Id: hal-01611595 \\ https://hal.science/hal-01611595}

Submitted on 6 Oct 2017

HAL is a multi-disciplinary open access archive for the deposit and dissemination of scientific research documents, whether they are published or not. The documents may come from teaching and research institutions in France or abroad, or from public or private research centers.
L'archive ouverte pluridisciplinaire HAL, est destinée au dépôt et à la diffusion de documents scientifiques de niveau recherche, publiés ou non, émanant des établissements d'enseignement et de recherche français ou étrangers, des laboratoires publics ou privés. 


\title{
Bending modulus of bidisperse particle rafts: Local and collective contributions
}

\author{
Pauline Petit and Anne-Laure Biance \\ Institut Lumière Matière, Université de Lyon, UMR 5306 Université Lyon 1-CNRS, 69622 Villeurbanne, France
}

Elise Lorenceau

Laboratoire Navier, Université Paris-Est, UMR 8205 CNRS, Ecole des Ponts ParisTech, IFSTTAR, 77420 Champs sur Marne, France

\author{
Carole Planchette* \\ Institute of Fluid Mechanics and Heat Transfer, Graz University of Technology, Austria \\ and Research Center Pharmaceutical Engineering GmbH, Graz, Austria
}

(Received 8 October 2015; published 11 April 2016)

\begin{abstract}
The bending modulus of air-water interfaces covered by a monolayer of bidisperse particles is probed experimentally under quasistatic conditions via the compression of the monolayer, and under dynamical conditions studying capillary-wave propagation. Simple averaging of the modulus obtained solely with small or large particles fails to describe our data. Indeed, as observed in other configurations for monodisperse systems, bidisperse rafts have both a granular and an elastic character: chain forces and collective effects must be taken into account to fully understand our results.
\end{abstract}

DOI: 10.1103/PhysRevE.93.042802

Particles adsorbing at liquid interfaces can stabilize interfaces in foams or Pickering emulsions [1-3] or encapsulate and protect isolated droplets and bubbles [4-6]. They constitute the key component of flotation where particles are sorted according to their hydrophobicity. Consequently, many fundamental studies have focused on the properties of these interfaces, noting, for example, the main role of interparticle interactions on raft crystalline phases [7-9]. Since the adsorbed particle monolayer can constitute a protection against coalescence, or can delay coarsening in multiscale materials, its mechanical properties have also been investigated extensively. For example, the influence of particle diameter on the monolayer collapse threshold [10], the interface bending [11,12], and the robustness against coalescence upon impact [4,13] have been determined. A complete description of these interfaces, granular by nature, must sometimes account for local elastic properties or overall parameters such as stress propagation in the media $[14,15]$.

Yet, while bidisperse particles are often assemble to increase the monolayer density, polydispersity effects have barely been considered. Surface pressure isotherm of bidisperse assemblies at liquid-liquid interfaces have been studied experimentally $[8,16]$, and they showed no difference with monodisperse systems. However, the bending ability, crucial for limiting coarsening, for example, remains unexplored. In this study, we characterize experimentally the density of bidisperse granular rafts, and we show how it affects their bending modulus, measured via static compression and propagation of surface waves. It appears that simple averaging of the properties obtained solely with the small or large particles cannot accurately describe our experimental results. Instead, both local individual properties of the adsorbed beads and collective effects must be considered.

To prepare the rafts, silanized spherical glass beads [12] with a density of $2500 \mathrm{~kg} \mathrm{~m}^{-3}$ and a contact angle $\theta=$

*carole.planchette@tugraz.at $107^{\circ} \pm 10$ are used. The beads are sifted mechanically to obtain monodisperse distributions with mean bead diameters ranging from 32 to $565 \mu \mathrm{m}$, and a standard deviation of $8 \%$. To achieve bidisperse distributions, the sifted particles are mixed in known proportion. The resulting mixtures are characterized by $d, D, n$, and $N$, the small- and large-particle diameters and numbers, respectively. Four particle size ratios are considered that correspond to seven samples for which the mean particle diameters are reported in Table I.

The particles are then gently deposited at the water interface in a few-cm-wide recipient by blowing air. A moving barrier allows us to adjust the laden interface area to the monolayer collapse threshold [11]. The proportion of small and large particles is measured using many top-view snapshots such as those shown in Fig. 1(a), and it is found to be the same as that in the samples. The monolayers are further characterized by their apparent surface density $\phi=\left(N \pi D^{2}+n \pi d^{2}\right) / 4 S$, with $S$ the investigated area. Variations of $\phi$ against the large particle fraction $\psi_{l}=N D^{2} /\left(n d^{2}+N D^{2}\right)$ are reported in Fig. 1(b) for different $d / D$.

For important diameter differences (samples 1 and 3), a maximum is observed for $\psi_{l} \approx 0.8$, while the curves remain flat for moderate ones (samples 5 and 7). The packing of continuous polydisperse assemblies, exhibiting, for example, Gaussian polydispersity, has been tackled extensively [17]. Yet, despite their wide use, noncontinuous distributions have been less studied. The problem has been investigated analytically and numerically in three dimensions [18,19] and numerically in two dimensions [20,21], but the results are not directly applicable to our systems. Indeed, since the particles lay at the water interface with a contact angle different from $\pi / 2$, they are not in contact at their equator, and our systems are neither two-dimensional (2D) nor 3D. Corrections to the simple 2D case are thus proposed, and the calculations are detailed in the Appendix.

Two cases are considered. For diameter ratios close to $1(d / D \sim 1)$, the monodisperse regime is achieved and the surface density is expected to be constant, equal to 
TABLE I. Sample number; $d$ and $D$ are the small- and largeparticle diameters, respectively.

\begin{tabular}{lccrrrrr}
\hline \hline Sample & 1 & 2 & 3 & 4 & 5 & 6 & 7 \\
\hline$d / D$ & 0.13 & \multicolumn{2}{c}{0.22} & \multicolumn{2}{c}{0.39} & \multicolumn{2}{c}{0.57} \\
$d(\mu \mathrm{m})$ & 72 & 32 & 128 & 32 & 128 & 83 & 72 \\
$D(\mu \mathrm{m})$ & 565 & 146 & 565 & 83 & 312 & 146 & 128 \\
\hline \hline
\end{tabular}

$\phi_{m}=\pi / 2 \sqrt{3} \simeq 0.9$, as observed for samples 5 and 7 in Fig. 1(b). The other one corresponds to low size ratios, where the small particles can partially fill the voids between large ones (samples 1 and 3). This effect is maximum if the small particles perfectly fill the voids. Taking $\theta=107^{\circ}$ as for our particles leads to $d / D \simeq 0.179$ (see the Appendix). In this case, two limits can be described analytically. When $\psi_{l}$ approaches 1 , the large particles form a dense network that occupies the maximum surface fraction of monodisperse systems $\phi_{m}$. The small particles fill the voids between the large ones. The surface fraction reads

$$
\phi=\phi_{m} / \psi_{l} \text {. }
$$

For $\psi_{l}=\frac{1}{2-\phi_{m}}$, the densely packed small particles exactly fill the gaps, and the maximum surface density, $\phi_{\max }=\phi_{m}\left(2-\phi_{m}\right)$, is reached. Surface density variations are slightly affected by the not truly $2 \mathrm{D}$ character of the rafts. Deviations are smaller than 0.03 (see the Appendix) and will be neglected thereafter. In contrast, if $\psi_{l}$ approaches zero, the raft is made of a dense packing of small particles having a surface fraction $\phi_{m}$, in which a few spots constituted of isolated large particles are found. The number $n_{r}$ of small (a)

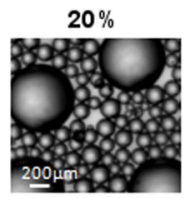

(b)

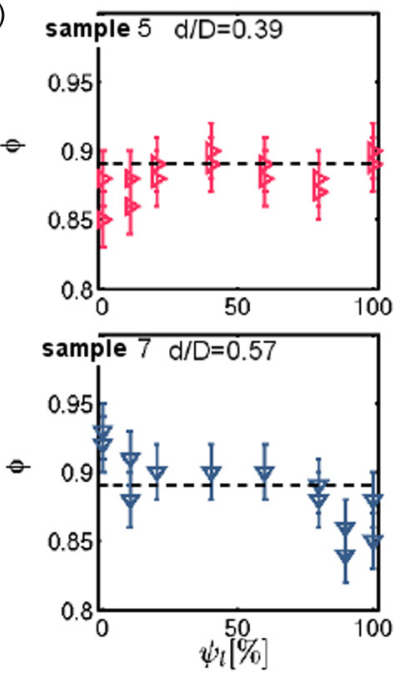

$40 \%$
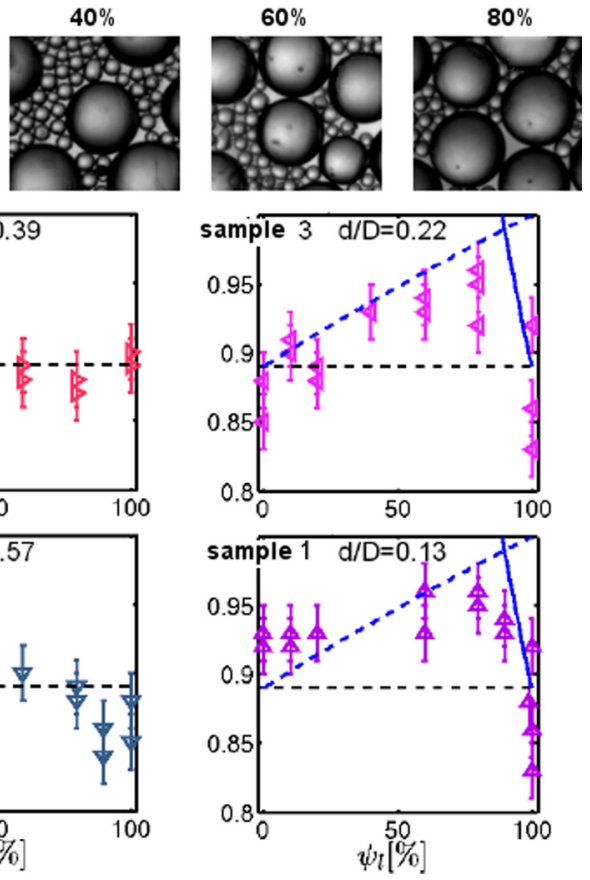

FIG. 1. (a) Particle monolayer snapshots (sample 3, Table I) for different $\psi_{l}$. (b) Surface density $\phi$ vs large particle fraction $\psi_{l}$ (symbols) for different samples (Table I). Plain lines correspond to Eq. (1), horizontal dashed lines to $\phi=\phi_{m}$, and tilted dashed lines to Eq. (2).

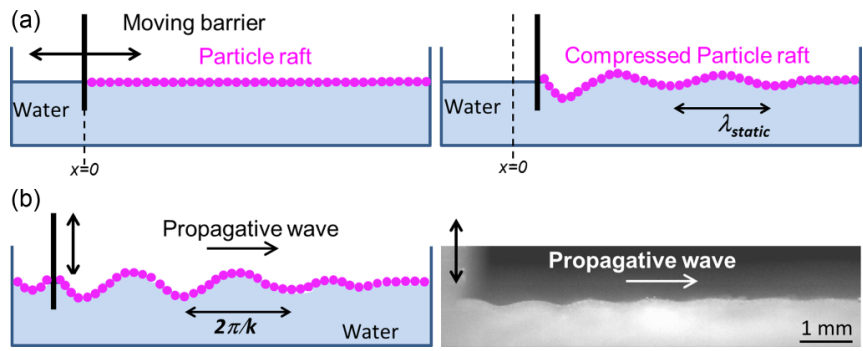

FIG. 2. (a) Scheme of the quasistatic assay producing wrinkles in the monolayer. (b) Left: Scheme of the dynamical assay for wave propagation. Right: wave propagation image (side view) used to obtain the dispersion relations.

particles replaced by large ones is $n_{r} \pi d^{2} / \phi_{m}=N \pi D^{2}$. The measured surface density reduces to

$$
\phi=\phi_{m} /\left[1-\psi_{l}\left(1-\phi_{m}\right)\right] \text {. }
$$

These predictions [Eqs. (1) and (2)] are compared to the experimental results in Fig. 1(b) for samples 1 and 3. They show very good agreement, confirming that the surface density is barely affected by the approximative 2D character of the raft.

Along with the description of raft surface density, we evaluate the effects of bidispersity on the raft-bending modulus. The first experimental setup, designed to probe quasistatic conditions, consists of a 6-cm-wide trough filled with deionized water, covered with silanized beads [11]. The confined monolayer is compressed by a moving barrier, as shown in Fig. 2(a). Wrinkles that appear above a certain compression are visualized via their shades under low-angled light. Both the overall compression and the local structure are recorded via top imaging.

For monodisperse rafts, we report in Fig. 3 the variations of $\lambda_{\text {static }}$, which is the wrinkle wavelength, with $d$ the particle diameter.

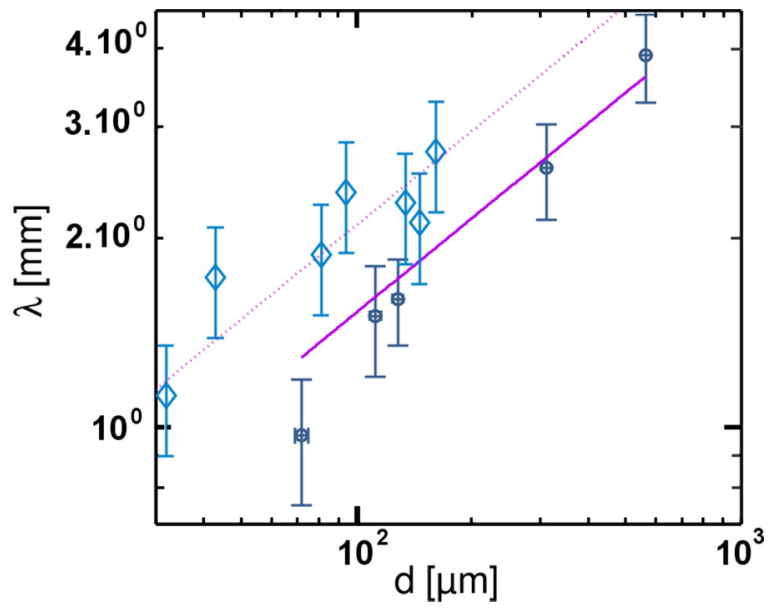

FIG. 3. (o), Quasistatic wavelengths obtained by quasistatic compression of monodisperse rafts as a function of the particle diameter $d .(\diamond)$, Equivalent dynamic wavelengths deduced from the dynamic bending modulus measured via capillary-wave propagation on monodisperse rafts as a function of the particle diameter $d$. The straight and dashed lines correspond to $\lambda \propto d^{1 / 2}$, in good agreement with the experimental points. 

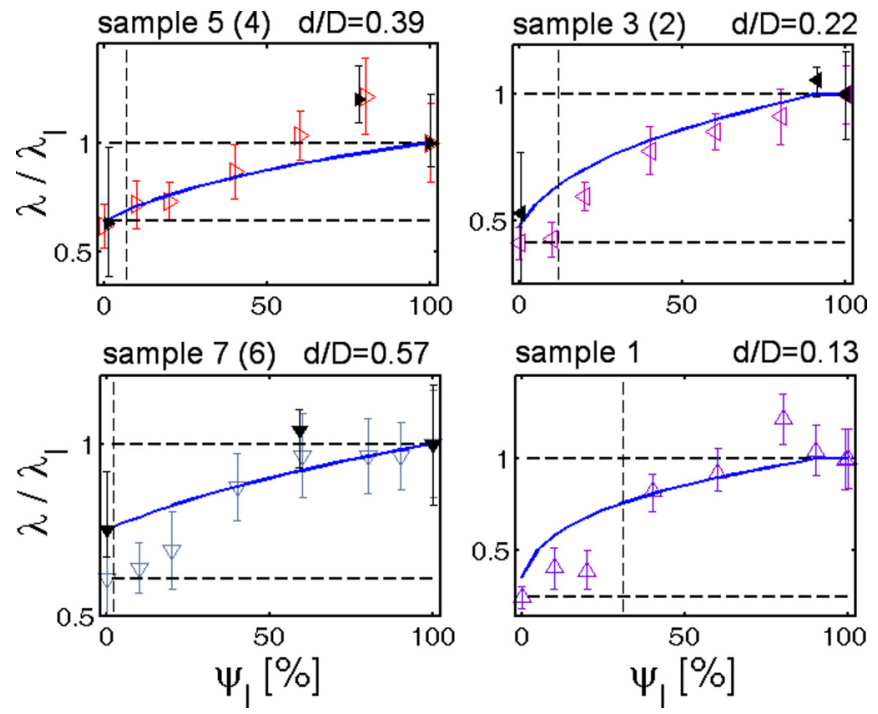

FIG. 4. Open symbols: wrinkles wavelength from quasistatic compression $\left(\lambda_{\text {static }}\right)$, normalized by the one of the large particles $\left(\lambda_{\text {static }, l}\right)$, for different $d / D$ (Table I). The error bars are standard deviations. The dashed horizontal straight lines represent the quasistatic wavelengths of monodisperse cases. The plain lines correspond to Eq. (3). Plain symbols: fourth root of the dynamic bending modulusobtained studying capillary-wave propagation-normalized by its value for large particles $\left(B_{\mathrm{dyn}} / B_{\mathrm{dyn}, l}\right)^{1 / 4}$, also referred to as $\lambda_{\mathrm{dyn}} / \lambda_{\mathrm{dyn}, l}$ for similar $d / D$ (Table I). The vertical dashed lines correspond to $\phi_{c}$ (see the text).

As for elastic plates or lipidic membranes, these wrinkles are attributed to a bending stiffness. Yet here, the bending modulus $B$ originates from wetting, and it has been shown that $\lambda_{\text {static }} \propto B^{1 / 4}$, where $B=\phi \sigma d^{2} f(\theta)$, with $\sigma$ the liquid surface tension, $d$ the particle diameter, and $f(\theta)$ a function depending on a geometrical factor and the contact angle $\theta[11,22,23]$. Our results obtained with monodisperse rafts, reported in Fig. 3 (o), are in good agreement with the commonly accepted scaling $\lambda_{\text {static }} \propto d^{1 / 2}$ except for the smallest particles, where we note some small deviation. This discrepancy may be attributed to the practical difficulties encountered to form a regular monolayer while using small particles.

To estimate the effect of bidispersity on raft-bending abilities, we report in Fig. 4 the observed wavelength normalized by that of the large particles $\left(\lambda_{\text {static }} / \lambda_{\text {static }, l}\right)$ as a function of $\psi_{l}$. The two values of large- and small-particle wavelengths ( $\lambda_{\text {static }, l}$ and $\left.\lambda_{\text {static, } s}\right)$ are recovered when $\psi_{l}$ tends to 1 and 0 , respectively. Moreover, one can observe that despite error bars, the wavelength can become larger than that of large particles alone; see Fig. 4, samples 1 and 5.

To confirm this feature and to investigate the effect of dynamical friction among the beads on the raft-bending abilities, capillary-wave propagation is considered [12]. A glass slide, fixed to a mechanical oscillator, is placed in contact with the monolayer as sketched in Fig. 2(b), transmitting its movements to the covered interface. Wave celerity, $c$, is recorded by side view imaging (4000 fps, phantom) for oscillation frequencies, $f$, ranging from 150 to $900 \mathrm{~Hz}$. The resulting wave number, $k$, is measured as a function of $f$. The experimental dispersion relation $c(k)$ with $c=2 \pi f / k$ is then constructed for different bidisperse rafts and fitted using the model and procedure described in [12] and in the Appendix. Following this approach, the dynamic bending modulus, $B_{\text {dyn }}$, is derived from the fitting procedure. To compare these results with those from quasistatic compression, the dynamic bending modulus is converted into an equivalent dynamic wavelength, $\lambda_{\text {dyn }}$, using the relation $\lambda_{\text {dyn }}=2 \pi\left(B_{\text {dyn }} / \rho g\right)^{1 / 4}, \rho$ being the liquid density, here water [11].

For monodisperse rafts, the behavior $\lambda_{\text {dyn }} \propto d^{1 / 2}$ is recovered (Fig. $3(\diamond))$. A discrepancy in the numerical factor is observed, however, between the static and dynamical experiments, as already found (but in the other way) before [12]. This highlights the fact that wetting is indeed a key ingredient to understand raft-bending ability, but that some other additional sources of interactions, such as dynamical interparticle friction or contact angle hysteresis, must be taken into account.

The results obtained on bidisperse rafts are compared to those of the quasistatic assay by plotting the values of $\left(B_{\mathrm{dyn}} / B_{\mathrm{dyn}, l}\right)^{1 / 4}$, corresponding to $\lambda_{\mathrm{dyn}} / \lambda_{\mathrm{dyn}, l}$ as a function of $\psi_{l}$ in Fig. 4 (plain symbols). Here, $B_{\mathrm{dyn}, l}$ corresponds to the measured value of $B_{\text {dyn }}$ for the large particles only, using the same method. In both cases, the error bars represent the variations resulting from the fitting procedure. Nevertheless, the dynamical results confirm that the bending modulus of bidisperse rafts can become larger than that found for rafts constituted of the large particles only (sample 4).

A natural way to compute the bending modulus of composite material composed of two materials of different moduli is to average the bending modulus obtained for each of the monodisperse rafts, as generally performed in predicting mechanical properties of composite materials [24].

The simplest approach, justified by energy arguments, consists in averaging according to surface density. Indeed, surface energy is a possible origin of the bending stiffness of the particle raft: assuming that there is no contact angle hysteresis, the particles at the interface independently self-adjust under bending of the interface to fulfill the condition of static contact angle. This induces surface energy variations proportional to the number of particles. Using the definitions of $\phi_{l}, \phi_{s}$, and $\psi_{l}$, it should read $B=\sigma f(\theta)\left(\phi_{l} D^{2}+\phi_{s} d^{2}\right)$, with $\phi_{s}$ and $\phi_{l}$ the surface fraction of small and large particles, respectively. Using $\lambda_{\text {dyn }} \propto B_{\text {dyn }}{ }^{1 / 4}, \lambda / \lambda_{l}$, the normalized wavelength (static or dynamic) of bidisperse mixtures reads

$$
\frac{\lambda}{\lambda_{l}}=\sqrt{\frac{d}{D}}\left(\frac{\phi}{\phi_{m}}\right)^{1 / 4}\left(1-\psi_{l}+\psi_{l} \frac{D^{2}}{d^{2}}\right)^{1 / 4} .
$$

Equation (3) (continuous lines) is compared, using Eqs. (1) and (2), with the experimental results in Fig. 4. Two main discrepancies can be identified. First, for small $\psi_{l}$, the bending modulus is systematically smaller than model predictions. This may not be explained solely by the deviation of the scaling $\lambda \propto d^{1 / 2}$ for the smallest particles $(d=72 \mu \mathrm{m})$ since this observation is also made for sample 3, whose small particles have a diameter of $128 \mu \mathrm{m}$. A possible origin of this deviation is the following: when the distance separating large particles is greater than the small-particle wavelength, the latter is expected to be recovered. Approximating $\phi$ by $\phi_{m}$, this threshold is reached for $\psi_{l}=\pi D^{2} / 2 \sqrt{3} \phi_{m} \lambda_{s}{ }^{2}=D^{2} / \lambda_{s}{ }^{2}$, where $\lambda_{s}$ is the wavelength measured for small particles only. Numerical application leads to $0.07,0.12,0.02$, and 0.31 for, 
respectively, samples $5,3,7$, and 1 . This is in agreement with our data, as can be seen in Fig. 4, where this threshold $\phi_{c}$ is highlighted using dashed vertical lines. On the other hand, for large $\psi_{l}$, the maxima observed in both static and dynamical measurements are not captured by Eq. (3). This suggests the need for a more complex modeling for this composite material.

To go further, two main routes are proposed. On the one hand, a more complex averaging of the two monodisperse moduli can be envisaged, as was explored extensively both theoretically and experimentally in continuum mechanics [24,25]. This would only be applicable for small particles to ensure that the continuity hypothesis holds. Moreover, it would require a full determination of the stress and strain in the media by knowing the material's constitutive equation. The latter requires a better local modeling of the forces between the particles, taking into account the full determination of the airwater interface deformation between the particles, the effect of contact angle hysteresis, and the potential heterogeneity of contact forces between particles of different sizes. On the other hand, in the limit of large particles, an alternative route consists in taking into account the granular character of these interfaces. Indeed, it has been shown that in truly 2D granular media, force chains build up between particles that distribute and propagate the stress within the media. The force chains are known to adopt specific directions in monodisperse systems that are fixed by the lattice and its orientation [26]. In nonmonodisperse systems exhibiting a relatively narrow size distribution, these chains lose their specific orientation due to the loss of order within the granular lattice, and they tend to disappear in favor of a more homogeneous stress distribution [26]. For a wider size distribution, Voivret et al. showed in a numerical study that the orientation of contact force chains follows the spatial distribution of the largest particles, which mediate most of the stress via their interactions [27]. In bidisperse systems, the order being lost, it is therefore expected that the selection of the contacts involved in force chains does not follow any specific orientation. Instead, the selection is driven by preferred particle interactions. In our systems, pair interactions can involve two particles of the same kind or one of each kind. Since to satisfy the contact angle with water, the large and small particles are not in the same plane (see the Appendix), it seems reasonable to consider that contacts between two particles of the same kind are more efficient to propagate forces and stress. As a result, when the system is percolated with the small particles, chain forces preferably build up between small particles only, thus bidisperse rafts can be seen as monodisperse rafts made of small particles with the inclusion of stiffer isolated areas of limited size where the large particles are found. The effective bending modulus of such composite materials is thus expected to be found between average values provided by Eq. (3) and the values given by small particles alone. In contrast, when the system is percolated with the large particles, its bending modulus is expected to take an intermediate value between that obtained for large particles only and that provided by the averaging approach. Numerical simulations evaluate the percolation threshold for $\psi_{l}$ between 0.5 and 0.6 [28]. This is in agreement with our observations, since for low $\psi_{l}$ the wavelength is below Eq. (3), while above $\psi_{l}=0.5$ all the measured wavelengths are equal to or larger than those of the large particles alone.
However, the existence of wavelengths greater than those of large particles alone cannot be captured by any of these interpretations. A plausible qualitative explanation is the formation of patterns whose elementary unit size exceeds that of large particles, as observed in curved geometries for monodisperse lattices.

In conclusion, the bending modulus of bidisperse particle rafts has been investigated by two distinct tests probing the raft quasistatic and dynamic responses. We propose to describe our data using two different frameworks. In the framework of continuum mechanics, we show that surface averaging of the moduli of small and large particles only produces a qualitative agreement. Indeed, it fails to accurately reproduce two experimental observations. First, the bending modulus of the bidisperse raft undergoes a relatively sharp transition for $\psi_{l} \approx 0.25-0.5$, which can only be explained in the framework of granular matter assuming that force chains are preferably mediated by contacts between particles of the same size. When the system is percolated with small particles, the bending modulus deviates from the averaging predictions toward the value corresponding to the monodisperse raft of small particles, and, by analogy, a similar deviation toward the bending modulus of the large-particle raft is observed when the system is percolated with large particles. The second observation, which could not be reproduced by our modeling, involves the existence for both quasistatic and dynamical measurements of maxima in the bending modulus that exceed the values obtained by large particles only. We propose to explain this observation by structural interactions with the formation of a lattice involving both small and large particles. It has yet to be determined how these results can be refined to predict the bending modulus maximum, and how they apply to wider particle distributions.

A.L.B. would like to thank T. Yanagashima for fruitful discussions. P.P. is thankful to RCPE for the financial support of her stay in Graz.

\section{APPENDIX}

\section{Apparent diameter}

In bidisperse rafts, an apparent particle radius can be defined that is different from the real radius since small- and largeparticle equators are not in the same plane. This concept is further used to correct our 2D analysis of raft solid surface fraction. Using a simple geometric argument, see Fig. 5, we determine the altitude $z_{t}$ at which the beads are touching each other.

At this altitude, the apparent diameters of the small sphere $d_{a}$ and the large sphere $D_{a}$ are given by (introducing $q=d / D$ )

$$
\begin{aligned}
& D_{a}=D \sqrt{1-\cos ^{2} \theta\left(\frac{q-1}{q+1}\right)^{2}}, \\
& d_{a}=d \sqrt{1-\cos ^{2} \theta\left(\frac{q-1}{q+1}\right)^{2}} .
\end{aligned}
$$

We can try to calculate the consequences of equator shift between large and small particles on the surface fraction of bidisperse rafts in the limit cases defined in the paper. When the two diameters are similar, $d / D \rightarrow 1$, the apparent diameter is close to the real one and no deviation is reported $\left(\phi \rightarrow \phi_{m}\right)$. 


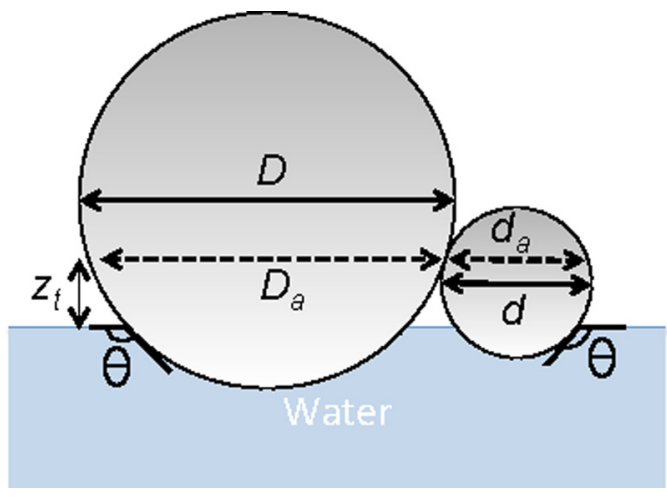

FIG. 5. Scheme of a side view of two particles laying on the water surface, with a contact angle $\theta$. The small and large particles touch each other at the altitude $z_{t}$, corresponding to their apparent diameter $d_{a}$ and $D_{a}$, respectively.

When the two diameters are different, $d / D \rightarrow 0$, deviations are then expected. In the first regime where $\psi_{l}$ is close to 1 (the great proportion of large particles), the large particles form a network of monodisperse particles having a surface fraction $\phi=\phi_{m}$. Within the voids, small particles can be inserted. As in the 2D case, the surface fraction is then given directly by definition of $\psi_{l}$ by $\phi=\phi_{m} / \psi_{l}$. In two dimensions, we expect that the maximum surface fraction is reached when the small-particle surface fraction is at random close packing in the void, i.e., when $\phi=\phi_{m}+\left(1-\phi_{m}\right) \phi_{m}$, which corresponds to $\psi_{l}=1 /\left(2-\phi_{m}\right)$. Deviations are expected, however, due to the $3 \mathrm{D}$ character of bidisperse rafts. Indeed, small particles have a larger area they can fill and can reach random close packing in the voids, based on the apparent diameter of the large particles. Thus, at the altitude $z_{t}$, the large particles cover a surface fraction given by $\phi_{l, t}=\phi_{m} D_{a}^{2} / D^{2}$. At their maximum coverage, the small particles will cover in this plane a surface fraction $\phi_{s, t}=\phi_{m}\left(1-\phi_{l, t}\right)$. So, at its maximum, the total surface fraction of the small particles is

$$
\phi_{s}=\phi_{s, t} \frac{d^{2}}{d_{a}^{2}}=\phi_{m}\left(1-\phi_{m} \frac{D_{a}^{2}}{D^{2}}\right) \frac{d^{2}}{d_{a}^{2}} .
$$

Denoting $K=\cos ^{2}(\theta)\left(\frac{q-1}{q+1}\right)^{2}$, the maximum surface fraction, in this case, is given by

$$
\phi=\frac{\phi_{m}}{1-K}\left[2-K-(1-K) \phi_{m}\right]
$$

corresponding to

$$
\psi_{l}=\frac{1-K}{2-K-(1-K) \phi_{m}} .
$$

If we compare these expressions to that of a pure 2D system $\left[\phi=\phi_{m} / \psi_{l}, \psi_{l}=1 /\left(2-\phi_{m}\right)\right]$, in the worst case of our experimental conditions, $K=0.04$. This generates a shift of surface fraction below 0.03 , which is smaller than our experimental incertitude.

Let us now consider the other limit $\psi_{l} \rightarrow 0$. In this case, the small particles create a network of surface fraction $\phi_{m}$. Some spots of large particles replace the small ones. The number of small particles replaced by large ones is given by $n_{r} \pi d_{a}^{2} / \phi_{m}=$ $N \pi D_{a}^{2}$. The measured surface density then satisfies

$$
\phi_{m}-\phi_{m} \phi \psi_{l}+\psi_{l} \phi=\phi
$$

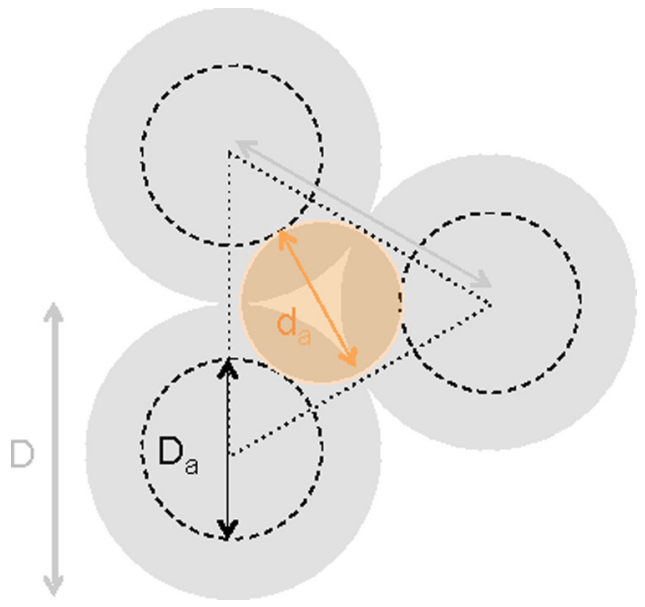

FIG. 6. Top view of three large particles touching each other. A small particle (bright, center) is inserted in the cavity formed by the three large particles.

so the surface density as defined here is not affected by this 3D geometry:

$$
\phi=\frac{\phi_{m}}{1+\phi_{m} \psi_{l}-\psi_{l}} .
$$

\section{Critical radius ratio $q$}

Considering the 3D geometry represented below, we look for the maximum diameter $d$ of a particle inserted in the void between three large particles of diameter $D$, densely packed (see Fig. 6). It is given by

$$
\frac{D_{a}+d_{a}}{D}=\frac{4}{3} \cos (\pi / 6)=\frac{2 \sqrt{3}}{3},
$$

which reduces to

$$
d / D=q=\frac{3+\cos (2 \theta)-\frac{4}{\sqrt{3}} \sqrt{2+\cos (2 \theta)}}{\cos (2 \theta)-1} .
$$

For $\theta=107^{\circ}$, this yields a critical radius ratio of 0,179 , very close to $2 / \sqrt{(} 3)-1 \simeq 0.154$ obtained for a truly $2 \mathrm{D}$ bidisperse configuration.

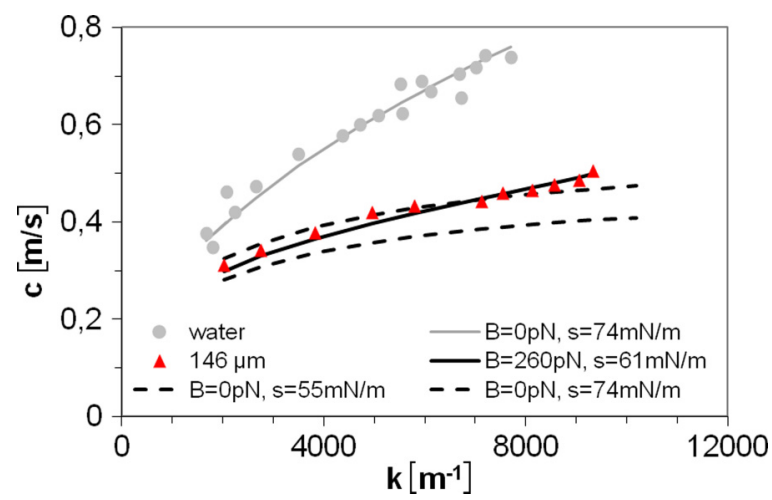

FIG. 7. Experimental dispersion relation of water and of a monodisperse raft of particles of $146 \mu$ m diameter. 


\section{Bending modulus from capillary-wave propagation}

To obtain the raft curvature modulus, $B$, the experimental dispersion relation is fitted by a modified wave propagation relation [13]. To limit the details, we simply reproduce the classical (A10) and modified (A11) equations,

$$
c^{2}=\frac{g}{k}\left(1+\frac{\sigma k^{2}}{\rho g}\right),
$$

with $c$ the wave celerity, $k$ the modulus of the wave vector, $\sigma$ the surface tension of the liquid, and $\rho$ its density. The modified relation reads

$$
c^{2}\left(1+\frac{\rho_{0}}{\rho} a k\right)=\frac{g}{k}\left(1+\frac{s k^{2}}{\rho g}+B \frac{k^{4}}{\rho g}\right) .
$$

To take into account the raft inertia, the monolayer average thickness $a$ and its density $\rho_{0}$ are introduced [Eq. (A11), second term on the left-hand side]. The surface tension of the bare liquid is replaced by an effective surface tension, $s$, since it is known that the presence of a particle monolayer reduces the surface tension of the interface [8,29]. The last term on the right-hand side accounts for the bending itself.

Using this theoretical expression, we adjust two parameters, $s$ and $B$, to fit the experimental data. Typical results obtained for pure water and a monodisperse raft are presented in Fig. 7. In contrast with pure water, the raft dispersion relation cannot be reproduced without introducing a bending modulus. The same procedure was applied to various monodisperse and bidisperse rafts, as illustrated in Fig. 8.
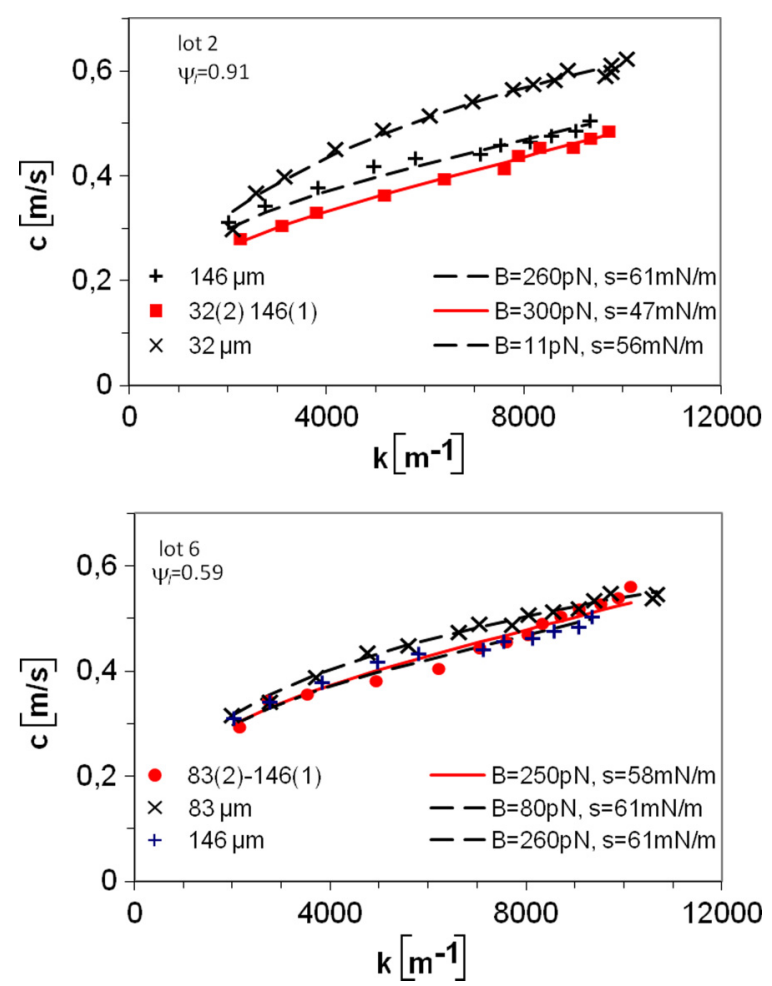

FIG. 8. Experimental dispersion relations (symbols) and corresponding fits (lines) of samples 2 and 6 with $\psi_{l}=0.91$ and 0.59 , respectively (red). The dispersion relations and fits of corresponding monodisperse rafts are also plotted (black).
[1] B. P. Binks, Particles as surfactants-Similarities and differences, Curr. Opin. Colloid Interface Sci. 7, 21 (2002).

[2] T. N. Hunter, R. J. Pugh, G. V. Franks, and G. J. Jameson, The role of particles in stabilising foams and emulsions, Adv. Colloid Interface Sci. 137, 57 (2008).

[3] B. P. Binks and R. Murakami, Phase inversion of particlestabilized materials from foams to dry water, Nat. Mater. 5, 865 (2006).

[4] P. Aussillous and D. Quere, Liquid marbles, Nature (London) 411, 924 (2001).

[5] P. Aussillous and D. Quere, Properties of liquid marbles, Proc. R. Soc. London, Ser. A 462, 973 (2006).

[6] M. Abkarian, A. B. Subramaniam, S. H. Kim, R. J. Larsen, S. M. Yang, and H. A. Stone, Dissolution Arrest and Stability of Particle-Covered Bubbles, Phys. Rev. Lett. 99, 188301 (2007).

[7] H. Xu, S. Melle, K. Golemanov, and G. Fuller, Shape and buckling transitions in solid-stabilized drops, Langmuir 21, 10016 (2005).

[8] C. Monteux, E. Jung, and G. G. Fuller, Mechanical properties and structure of particle coated interfaces: Influence of particle size and bidisperse 2D suspensions, Langmuir 23, 3975 (2007).

[9] E. L. Sharp, H. Al-Shehri, T. S. Horozov, S. D. Stoyanov, and V. N. Paunov, Adsorption of shape-anisotropic and porous particles at the air-water and the decane-water interface studied by the gel trapping technique, Rsc Adv. 4, 2205 (2014).

[10] S. Razavi, K. D. Cao, B. Lin, K. Y. C. Lee, R. S. Tu, and I. Kretzschmar, Collapse of particle-laden interfaces under compression: Buckling vs. particle expulsion, Langmuir 31, 7764 (2015).

[11] D. Vella, P. Aussillous, and L. Mahadevan, Elasticity of an interfacial particle raft, Europhys. Lett. 68, 212 (2004).

[12] C. Planchette, E. Lorenceau, and A. L. Biance, Surface wave on a particle raft, Soft Matter 8, 2444 (2012).

[13] C. Planchette, A. L. Biance, and E. Lorenceau, Transition of liquid marble impacts onto solid surfaces, Europhys. Lett. 97, 14003 (2012).

[14] P. Cicuta, E. J. Stancik, and G. G. Fuller, Shearing or Compressing a Soft Glass in 2d: Time-Concentration Superposition, Phys. Rev. Lett. 90, 236101 (2003).

[15] P. Cicuta and D. Vella, Granular Character of Particle Rafts, Phys. Rev. Lett. 102, 138302 (2009).

[16] H. Ma, B. Perea, and L. L. Dai, Study of two-component colloidal particles at air/water interfaces using langmuir-blodgett techniques, Coll. Surf. A 372, 61 (2010).

[17] C. Voivret, F. Radjai, J. Y. Delenne, and M. S. El Youssoufi, Space-filling properties of polydisperse granular media, Phys. Rev. E 76, 021301 (2007). 
[18] C. C. Furnas, Grading aggregates; mathematical relations for beds of broken solids of maximum density, Ind. Eng. Chem. 23, 1052 (1931).

[19] H. J. H. Brouwers, Packing of crystalline structures of binary hard spheres: An analytical approach and application to amorphization, Phys. Rev. E 76, 041304 (2007).

[20] N. Xu, J. Blawzdziewicz, and C. S. O'Hern, Random close packing revisited: Ways to pack frictionless disks, Phys. Rev. E 71, 061306 (2005).

[21] K. W. Desmond and E. R. Weeks, Random close packing of disks and spheres in confined geometries, Phys. Rev. E 80, 051305 (2009).

[22] R. Aveyard, J. H. Clint, and T. S. Horozov, Aspects of the stabilisation of emulsions by solid particles: Effects of line tension and monolayer curvature energy, Phys. Chem. Chem. Phys. 5, 2398 (2003).

[23] P. A. Kralchevsky, I. B. Ivanov, K. P. Ananthapadmanabhan, and A. Lips, On the thermodynamics of particle-stabilized emulsions: Curvature effects and catastrophic phase inversion, Langmuir 21, 50 (2005).

[24] R. M Jones, Mechanics of Composite Materials (McGraw-Hill, New York, 1975), Vol. 1.

[25] Z. Hashin and S. Shtrikman, A variational approach to the theory of the elastic behavior of multiphase materials, J. Mech. Phys. Solids 11, 127 (1963).

[26] J. F. Geng and R. R. Behringer, Slow drag in two-dimensional granular media, Phys. Rev. E 71, 011302 (2005).

[27] C. Voicret, F. Radjai, J.-Y. Delenne, and M. S. El Youssoufi, Multiscale Force Networks in Highly Polydisperse Granular Media, Phys. Rev. Lett. 102, 178001 (2009).

[28] P. H. Winterfeld, L. E. Scriven, and H. T. Davis, Percolation and conductivity of random two-dimensional composites, J. Phys. C 14, 2361 (1981).

[29] G. Lagubeau, Interfaces à grains et autres situations de mouillage nul, Ph.D. thesis, Université Pierre et Marie Curie, Paris, France, 2010. 